\title{
High tau levels in cerebrospinal fluid predict nursing home placement and rapid progression in Alzheimer's disease
}

\author{
Malin Degerman Gunnarsson ${ }^{1 *}$, Martin Ingelsson ${ }^{1}$, Kaj Blennow ${ }^{2}$, Hans Basun ${ }^{1}$, Lars Lannfelt ${ }^{1}$ and Lena Kilander ${ }^{1}$
}

\begin{abstract}
Background: Increased concentrations of cerebrospinal fluid (CSF) total tau (t-tau) and phosphorylated tau, as well as decreased amyloid- $\beta 42$ peptide, are biomarkers of Alzheimer's disease (AD) pathology, but few studies have shown an association with AD progression rate. We hypothesized that high CSF tau, as a marker of ongoing neurodegeneration, would predict a more aggressive course of AD, using time to nursing home placement (NHP) as the main outcome.
\end{abstract}

Methods: Our sample inlcuded 234 patients with mild cognitive impairment (MCI) due to AD $(n=134)$ or mild to moderate AD ( $n=100)$ who underwent lumbar puncture at a memory clinic and were followed for 2-11 years (median 4.9 years).

Results: Individuals with CSF t-tau in the highest quartile ( $\geq 900 \mathrm{ng} / \mathrm{L}$ ) had a higher ratio of NHP, both in the total cohort and in patients with MCl only (adjusted HR 2.17 [95 \% Cl 1.24-3.80]; HR 2.37 [95 \% Cl 1.10-5.09], respectively), than the lowest quartile. The association between high t-tau levels and future steep deterioration was confirmed in analyses with conversion to moderate dementia (HR 1.66; $95 \%$ Cl 1.08-2.56), rapid decline in Mini Mental State Examination score ( $\geq 4$-point drop/12 months), and dying in severe dementia as outcomes.

Conclusions: To our knowledge, this is the first study to show that high CSF t-tau levels predict early NHP and conversion to moderate dementia in an AD cohort. Selecting patients with high CSF t-tau, indicating more aggressive neurodegeneration and steeper decline, for AD immunotherapy trials might increase the possibility of showing contrast between active treatment and placebo.

Keywords: Nursing home placement, NHP, Alzheimer's disease, CSF, Tau, p-tau, Amyloid- $\beta$, Rapid decline, Moderate dementia, Death in severe dementia

\section{Background}

Alzheimer's disease (AD) is a progressive neurodegenerative disorder and the most common cause of dementia. It is characterized by a gradual cognitive deterioration, loss of function in activities of daily living (ADL), poor quality of life, and shorter survival [1]. At the stage of mild cognitive impairment $(\mathrm{MCI})$, patients are still autonomous. Subsequently, mild dementia is followed by moderate dementia, defined as loss of ability to perform personal activities of daily living (PADL). Further disease progression increases the burden on both patients and caregivers and

\footnotetext{
* Correspondence: malin.degerman.gunnarsson@pubcare.uu.se ${ }^{1}$ Department of Public Health and Caring Sciences/Geriatrics, Uppsala University, Uppsala Science Park, SE-751 85 Uppsala, Sweden Full list of author information is available at the end of the article
}

eventually leads to nursing home placement (NHP), with a tremendous impact on societal costs [2]. The rate of progression is highly variable between patients, supposedly reflecting the intensity of the neurodegenerative process $[3,4]$. It is of utmost importance to learn more about predictors of rapid decline in order to develop treatment aimed at slowing down the disease process.

Aggregation of misfolded amyloid- $\beta$ (A $\beta)$ is an early pathogenic event in AD. It is believed to mediate the formation of abnormally phosphorylated tau in neurofibrillary tangles [5]. These two processes are reflected by decreased amyloid- $\beta 42$ peptide $(\mathrm{A} \beta 42)$ and increased phosphorylated tau ( $\mathrm{p}$-tau) in the cerebrospinal fluid (CSF) [6], while high CSF total tau (t-tau) is a marker 
of ongoing axonal degeneration [7, 8]. These three biomarkers are predictors of conversion from the MCI stage to $\mathrm{AD}$ mild dementia; that is, they separate $\mathrm{MCI}$ due to $\mathrm{AD}$ from $\mathrm{MCI}$ non-AD cases on a group level $[9,10]$. However, in patients with established AD, levels remain stable from the prodromal/MCI stage onward [11]. Whether the magnitude of CSF t-tau may predict future speed of progression later in $\mathrm{AD}$ is less well understood.

Recent findings suggest that tau pathology can propagate between neurons [12, 13], and immunotherapy trials targeting tau have recently started [14]. We previously reported that high CSF tau can predict rapid cognitive decline and risk of dying in patients with severe dementia [15]. The aim of the present study was to investigate whether high CSF tau levels also would predict time to NHP. Factors known to promote early institutionalization, such as single living, education level, and baseline severity of cognitive impairment were taken into account. Further, we analyzed whether high CSF tau was related to three other proxies of progression rate: time to conversion to moderate dementia, rapid cognitive decline as measured by repeated evaluation with the Mini Mental State Examination (MMSE) [16], and risk of dying in patients with severe dementia.

\section{Methods}

\section{Study population and diagnostic procedures}

We conducted a retrospective study, and the cohort consisted of all patients referred to the Memory Clinic at the Uppsala University Hospital, where all five authors work as physicians. Participants underwent a lumbar puncture (LP) as part of the diagnostic procedure between 2003 and 2011 and were diagnosed with AD $(n=234)$. Computed tomography or magnetic resonance imaging scans, caregiver interviews, thorough cognitive assessments, and in some cases regional glucose uptake by positron emission tomography were other parts of the diagnostic workup. The diagnosis of probable $\mathrm{AD}$ dementia was set according to the National Institute of Neurological and Communicative Disorders and Stroke/ Alzheimer's Disease and Related Disorders Association criteria [17] and the Diagnostic and Statistical Manual of Mental Disorders, Fourth Edition, criteria [18]. Patients with neuroimaging findings suggesting a mixture of $\mathrm{AD}$ and cerebrovascular pathology were not included.

CSF samples were collected by LP in polypropylene tubes following standardized procedures. Samples with clear visual blood contamination were excluded. All CSF samples were centrifuged and aliquoted in polypropylene tubes, and all samples were frozen once before analyses, which were done continuously as part of routine clinical practice. CSF concentrations of $\mathrm{t}$-tau, $\mathrm{p}$-tau, and $\mathrm{A} \beta 42$ were determined using sandwich enzyme-linked immunosorbent assays (ELISAs) at the Clinical Neurochemistry Laboratory, University of Gothenburg, Mölndal, Sweden
$[8,19,20]$. Assays were performed by experienced, boardcertified laboratory technicians strictly following procedures approved by the Swedish Board for Accreditation and Conformity Assessment. To reduce variability over time and between batches, strict laboratory procedures were followed, including regular calibration of pipettes and preventive service of instruments (ELISA readers). For each assay, specified acceptance criteria for the calibration curve of an ELISA plate were applied for approval of each individual run, including optical density (OD) of the blank $<0.1$, OD of the highest calibrator between 1.2 and 3.0, OD of lowest calibrator larger than OD of the blank, and coefficient of variance in percent below $15 \%$ for each calibrator (run in duplicate). In addition, internal quality control (QC) samples consisting of aliquots of pooled CSF (with low and high biomarker levels) were run on each ELISA plate together with patient CSF samples. The QC samples were placed at the beginning and the end of each ELISA plate, and had to fall within a prespecified target value set by repeated analyses for each ELISA format. Last, to ensure conformance between ELISA batches, a batch-bridging procedure to compare old with new batches was performed using a panel of individual $(n>20)$ CSF samples. If a systematic difference was identified, the new batch was rejected and another batch was evaluated.

The CSF biomarker results were taken into account in the diagnostic procedure, together with all other information. Ninety-eight percent of the patients had at least one CSF biomarker outside the normal range. The original diagnoses were continuously reevaluated at follow-up visits for a minimum of 2 years, and all diagnostic workup was scrutinized by one of the authors (MDG) to ensure accuracy. Mild dementia was defined as impaired performance in instrumental ADL only, and moderate dementia was defined as loss of function in PADL (i.e., needing assistance with either of grooming, bathing, dressing, toileting, feeding, or ambulation). One hundred patients fulfilled the $\mathrm{AD}$ dementia criteria at baseline, and one hundred thirty-four individuals were at the MCI stage [21] at baseline and converted to AD dementia during follow-up. These 234 patients formed the study population and were followed for a maximum of 11 years to May 2014 or to the date of death.

\section{Outcomes}

The digital medical record system kept at the Uppsala County Council is common to all health care provided in this area, including the vast majority of the general practitioners (GPs) at the health centers and dementia group living facilities, which enabled follow-up from diagnosis to death. Most patients were followed at the Memory Clinic every 6-12 months during the first years after diagnosis, and they were subsequently followed at health centers and eventually at the nursing homes. 
Dates of moving to a nursing home were captured from the records, as were information on education level and living conditions. Descriptions of the patients' ADL function, cognitive deterioration, and medical status, as documented by the physicians at the Memory Clinic, by the GPs, and in the nursing homes also by registered nurses or occupational therapists, were collected from the medical records. Date of conversion to moderate dementia was defined as the date of the first documentation of impaired ability to perform PADL. Baseline MMSE scores were retrieved from the testing that most closely corresponded to the time of LP (within 1-3 months). Since this was a clinical study, the intervals of the clinical follow-up and MMSE retesting after baseline varied according to the individual patient's needs. Decline in MMSE was calculated as the difference between the baseline score and the last available test score divided by time in months, and we defined rapid cognitive decline as a $\geq 4$-point decline in MMSE per 12 months. Nine patients had a shorter time span than 6 months between baseline and last MMSE. Dying in severe dementia was identified as death after a prolonged decline over months at the end stage of $\mathrm{AD}$ and being immobilized and dependent in all PADL, in contrast to premature death due to other causes.

\section{Statistics}

Cox proportional hazards models were used to assess the HRs for NHP, conversion to moderate dementia, and dying in severe dementia. Eight individuals lacked sufficient information to determine cause of death and were censored as nonresponders at the date of death. Another 11 patients moved from Uppsala County Council and were censored as nonresponders for conversion to moderate dementia/NHP when they moved and as nonresponders at the date of death. Patients lacking MMSE scores at follow-up $(n=21)$ were withdrawn from the analyses of rapid decline. ORs of rapid cognitive decline were determined by logistic regression. All analyses were performed in crude models and the same multivariate models, adjusted for age, sex, living condition, education level, baseline mild to moderate dementia stage, and MMSE score. MCI was referenced when the models were adjusted for mild to moderate dementia. In addition, separate analyses were conducted with subjects with baseline mild to moderate dementia and $\mathrm{MCI}$ due to $\mathrm{AD}$, respectively. The CSF biomarker levels in univariate models and in stratified analyses $(\mathrm{MCI}$ and mild to moderate dementia) for conversion from mild to moderate dementia were entered with a split at median. In all other analyses, the CSF biomarker levels were entered as quartiles. Student's $t$ test was used for comparing normally distributed variables between groups. The level of statistical significance was set to $p=0.05$. All statistics and figures were produced using Statistica version 10 software (StatSoft, Tulsa, OK, USA).
Table 1 Description of the study population

\begin{tabular}{ll}
\hline Variable & Data \\
\hline$n$ & 234 \\
Follow-up period, years, median (range) & $4.9(2-11)$ \\
Age, years, median (range) & $70(46-86)$ \\
MMSE score, mean \pm SD & $24 \pm 5$ \\
Female sex & $145(62)$ \\
Coresidency status & \\
$\quad$ Cohabiting (female/male) & $99(68) / 79(89)$ \\
$\quad$ Living alone (female/male) & $46(32) / 10(11)$ \\
Education (years) & $114(49)$ \\
$\leq 7$ & $71(30)$ \\
$8-12$ & $49(21)$
\end{tabular}

CSF biomarkers $^{a}$ according to baseline AD stage, mean \pm SD

t-tau, ng/L

$\mathrm{MCl} 723 \pm 354$

p-tau, ng/L dementia $780 \pm 558$

$\mathrm{A} \beta 42, \mathrm{ng} / \mathrm{L}$

$\mathrm{MCl} 102 \pm 43$ dementia $102 \pm 48$

$\mathrm{MCl} 351 \pm 114$

All three biomarkers normal ${ }^{\mathrm{a}} \quad 5(2)$

Low Aß42, normal t-tau and p-tau 21 (9)

Low A 42 , high t-tau or p-tau 48 (21)

Normal Aß42, high t-tau and/or 29 (12)

p-tau

All three biomarkers pathological

$131(56)$

Baseline AD stage, $n$ (\%); MMSE score, mean (SD)

$\mathrm{MCl}$

134 (57); $26 \pm 3$

Mild dementia

85 (36); $22 \pm 4$

Moderate dementia

$15(7) ; 15 \pm 6$

NHP during follow-up

112 (48)

Males/females

$34(38) / 78$ (54)

$\mathrm{MCl} /$ dementia stage at baseline

$54(40) / 58(58)$

149 (68)

$83 / 134(62)$

From $\mathrm{MCl}$ at baseline

66/86 (77)

From mild dementia at baseline

57 (24)

Rapid cognitive decline ${ }^{b}$

$22(16)$

$\mathrm{MCl}$

35 (35)

Mild to moderate dementia

83 (35)

Death during follow-up

$46(20)$

Death in severe dementia during follow-up

19 (14)

$\mathrm{MCl}$

$27(27)$

A 442 amyloid- $\beta 42$ peptide, AD Alzheimer's disease, CSF cerebrospinal fluid, $\mathrm{MCl}$ mild cognitive impairment, MMSE Mini Mental State Examination, NHP nursing home placement, $\mathrm{p}$-tau phosphorylated tau, $\mathrm{t}$-tau total tau Values are number (percent) unless otherwise stated

${ }^{a}$ Reference values: $A \beta_{42}>450 \mathrm{ng} / \mathrm{L}, \mathrm{t}$-tau $<400 \mathrm{ng} / \mathrm{L}, \mathrm{p}$-tau $<80 \mathrm{ng} / \mathrm{L}$

${ }^{b} \geq 4$-point decline in MMSE per 12 months 

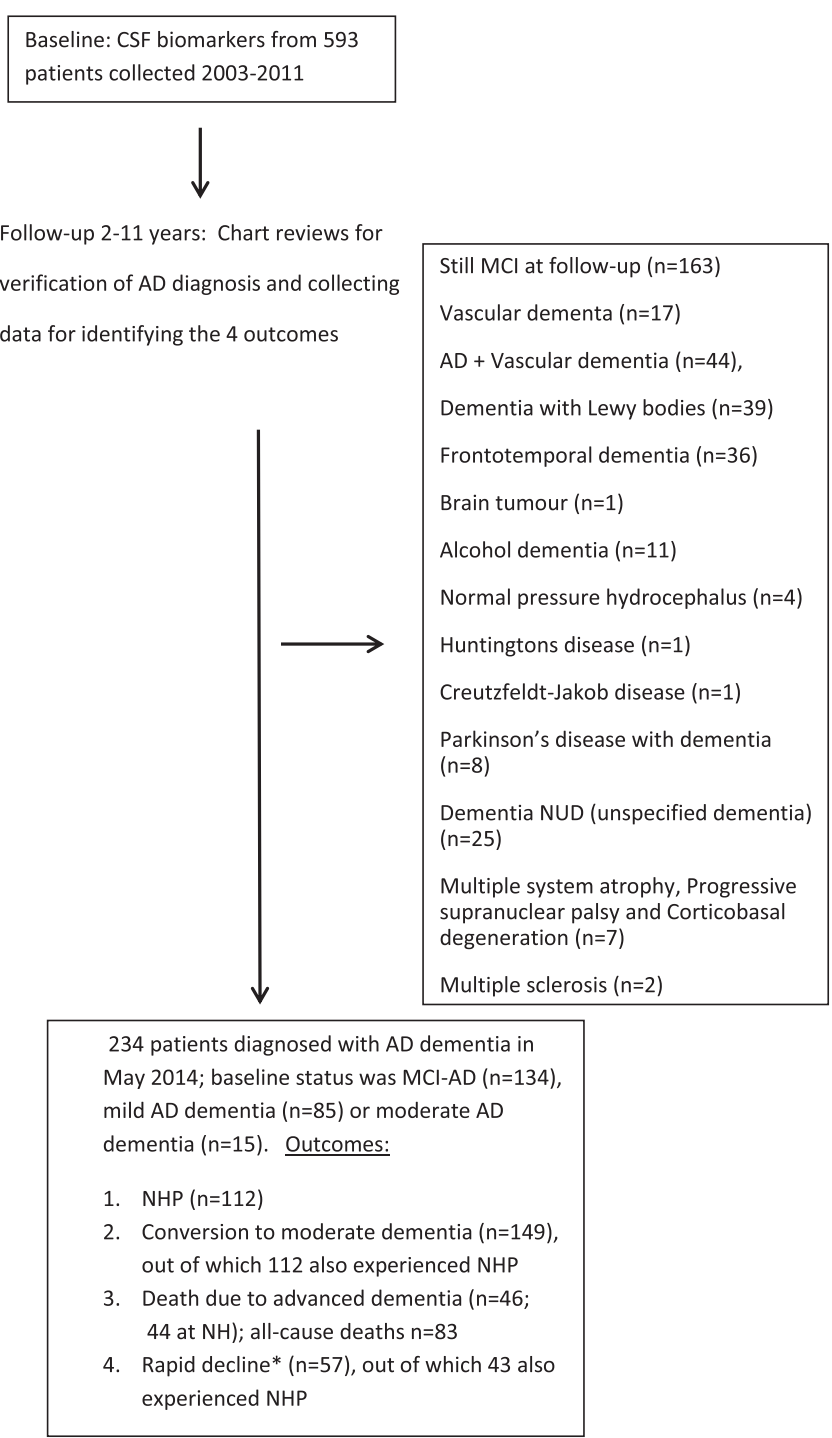

CSF = cerebrospinal fluid, $\mathrm{AD}=$ Alzheimer's disease, $\mathrm{MCl}=$ mild cognitive impariment, $\mathrm{NH}=$ Nursing home, NHP = Nursing home placement, ${ }^{*}$ Rapid decline $=\geq 4 \mathrm{ploss} / 12$ months in the Mini Mental State Examination

Fig. 1 Flowchart of the study population. CSF cerebrospinal fluid, AD Alzheimer's disease, MCI mild cognitive impairment, NH nursing home, NHP nursing home placement, NUD unspecified dementia

\section{Results}

Baseline demographics and clinical characteristics are shown in Table 1, and a flowchart is shown in Fig. 1. Concentrations of CSF biomarkers did not differ between the disease stages. All but nine patients were treated with cholinesterase inhibitors and/or memantine during part of the follow-up period. Apolipoprotein $\mathrm{E}$ genotypes were available for only 82 individuals $(\varepsilon 4 / 4, n=25 ; \varepsilon 4 / 3, n=39$; $\varepsilon 4 / 2, n=1$; $\varepsilon 4$ noncarriers, $n=17$ ), who were therefore not included in the analyses. During follow-up, 149 patients converted to moderate dementia and 112 patients were admitted to NHP; all had converted to moderate dementia before this event. The times from baseline to NHP (mean $\pm \mathrm{SD})$ were $4.3 \pm 1.9$ years in the MCI group $(n=54), 3.1$ \pm 1.3 years in the mild dementia group $(n=46)$, and $1.3 \pm$ 1.0 years in the moderate dementia group $(n=12)$.

Baseline CSF t-tau greater than the median value was associated with a higher risk of NHP (Table 2), with a dose-dependent relationship in crude and adjusted models (Table 3, Fig. 2). Individuals in the highest quartile of CSF t-tau ( $\geq 900 \mathrm{ng} / \mathrm{L})$ experienced the highest risk, which could also be seen when including subjects with MCI only (adjusted HR 2.37, 95 \% CI 1.10-5.09). The corresponding risk for patients with baseline mild AD was HR 3.11 (95 \% CI 1.35-7.13) in crude analyses, which did not reach statistical significance in the multivariate model. High p-tau 
Table 2 Cox proportional hazards ratios of nursing home placement, conversion to moderate dementia, and death in severe dementia during follow-up and ORs of rapid cognitive decline in univariate analyses

\begin{tabular}{|c|c|c|c|c|}
\hline Variable & $\begin{array}{l}\operatorname{NHP}(n=112 / 234) \\
\operatorname{HR}(95 \% \mathrm{Cl})\end{array}$ & $\begin{array}{l}\text { Conversion to moderate dementia } \\
(n=149 / 219), \mathrm{HR}(95 \% \mathrm{Cl})\end{array}$ & $\begin{array}{l}\text { Rapid cognitive decline }{ }^{\mathrm{a}} \\
(n=57 / 213), \text { OR }(95 \% \mathrm{Cl})\end{array}$ & $\begin{array}{l}\text { Death in severe dementia } \\
(n=46 / 234), \mathrm{HR}(95 \% \mathrm{Cl})\end{array}$ \\
\hline Age (per year) & $1.00(0.97-1.03)$ & $0.99(0.96-1.01)$ & $1.00(0.95-1.04)$ & $1.00(0.96-1.04)$ \\
\hline Male sex & $0.56(0.37-0.83)^{b}$ & $0.93(0.67-1.52)$ & $0.44(0.22-0.89)^{b}$ & $0.65(0.34-1.22)$ \\
\hline Cohabiting & $0.78(0.52-1.18)$ & $1.52(1.01-2.30)^{b}$ & $1.37(0.63-3.00)$ & $1.10(0.54-2.22)$ \\
\hline \multicolumn{5}{|l|}{ Education level } \\
\hline$\leq 7$ years & 1.00 & 1.00 & 1.00 & 1.00 \\
\hline $8-12$ years & $0.74(0.48-1.15)$ & $0.98(0.67-1.44)$ & $2.03(1.06-3.88)^{b}$ & $0.98(0.51-1.89)$ \\
\hline$\geq 13$ years & $0.38(0.22-0.65)^{b}$ & $0.76(0.50-1.16)$ & $0.43(0.17-1.09)$ & $0.27(0.10-0.63)^{b}$ \\
\hline $\begin{array}{l}\text { Mild to moderate dementia at } \\
\text { baseline }\end{array}$ & $1.74(1.20-2.53)^{b}$ & $1.71(0.85-3.44)$ & $2.99(1.57-5.69)^{b}$ & $3.25(1.80-5.86)^{b}$ \\
\hline MMSE at LP (per point) & $0.86(0.83-0.90)^{b}$ & $0.87(0.87-0.91)^{b}$ & $0.95(0.88-1.02)$ & $0.89(0.85-0.93)^{b}$ \\
\hline t-tau above median & $1.81(1.25-2.64)^{b}$ & $1.86(1.35-2.58)^{b}$ & $2.56(1.34-4.88)^{b}$ & $1.73(0.95-3.17)$ \\
\hline p-tau above median & $1.12(0.77-1.62)$ & $1.35(0.98-1.88)$ & $1.63(0.86-3.07)$ & $1.26(0.69-2.28)$ \\
\hline Aß42 below median & $1.35(0.93-1.97)$ & $1.21(0.88-1.67)$ & $1.05(0.56-1.97)$ & $1.31(0.76-2.37)$ \\
\hline
\end{tabular}

$A \beta 42$ amyloid- $\beta 42$ peptide, LP lumbar puncture, MMSE Mini Mental State Examination, NHP nursing home placement, $p$-tau phosphorylated tau, $t$-tau total tau $a^{a}$-point decline in MMSE per 12 months ${ }^{\mathrm{b}} p<0.05$

or low $\mathrm{A} \beta 42$ concentrations were not associated with NHP (Table 2, Additional file 1: Table S1, and Additional file 2: Table S2).

Similarly, subjects in the highest quartile of t-tau had higher rates of conversion to moderate dementia (Table 3, Fig. 3). CSF t-tau above the median was associated with increased rate of conversion to moderate dementia, both in MCI (adjusted HR 1.66, 95 \% CI 1.08-2.56) and in mild dementia (adjusted HR 1.80, 95 \% CI 1.02-3.20). These associations remained in crude and multivariate models including only cohabiting patients. There were no consistent associations between CSF A 342 or p-tau concentrations and this outcome in crude or adjusted models (Table 2, Additional file 1: Table S1, and Additional file 2: Table S2).

Our previous findings in 196 subjects of this cohort, followed for a shorter period [15], were confirmed in this extended population. Setting the lowest quartile of CSF ttau as a reference, adjusted OR for rapid cognitive decline in the highest quartile was 3.04 (95\% CI 1.16-8.99) (Table 3). Using a cutoff of MMSE $\geq 3$ points/year, the OR in patients with CSF t-tau above the median was 2.76 (95\% CI 1.465.21), MMSE $\geq 4$ points/year was 2.87 (95\% CI 1.41-5.84), and MMSE $\geq 5$ points/year was 2.35 (95\% CI 1.15-4.80) in the multivariate model. Patients in the highest $\mathrm{t}$-tau quartile also had a higher risk of dying in severe dementia (adjusted HR 2.64, 95 \% CI 1.10-6.33) (Table 3). Further, subjects in the highest quartile of p-tau had an increased HR of death in severe dementia compared with participants in the three lowest quartiles (adjusted HR 1.88, 95 \% CI 1.03-3.45); otherwise, p-tau was not significantly related to these outcomes (Additional file 1: Table S1). CSF A 342 was associated with neither rapid cognitive decline nor death in severe dementia (Additional file 2: Table S2). None of the CSF biomarkers was related to all-cause mortality.

\section{Discussion}

To our knowledge, this is the first report that a high CSF concentration of $\mathrm{t}$-tau predicts an earlier need of institutionalization (NHP) and conversion to moderate AD dementia, two solid markers of disease burden and societal costs. These associations were dose-dependent and present already at the stage of MCI due to AD. Moreover, they were independent of age, sex, education level, and living conditions as well as of baseline cognitive and functional status. We were able to capture these two outcomes in a memory clinic cohort through a long follow-up with virtually no loss. Further, subjects with AD who had high CSF t-tau had a steeper cognitive decline and a higher risk of dying in severe dementia, which confirms and expands previous reports $[15,22,23]$. In our previous study [14], CSF levels of p-tau were associated with an increased risk of rapid cognitive decline. In the present study, CSF A $\beta 42$ and p-tau showed no consistent associations with the rate of decline.

Our results fit well with the current pathophysiological model, where decreased A $\beta 42$ in CSF reflecting amyloid accumulation in the brain is a very early event, which is stable from the presymptomatic stage onward [24]. The density of amyloid plaques at autopsy is poorly correlated with clinical status, whereas there is a closer correlation between the extent of tangles and cognitive dysfunction [25, 26]. CSF t-tau and p-tau have been shown to increase already in the asymptomatic stage or 
Table 3 Crude and adjusted Cox proportional hazards ratios (95\% Cl) of nursing home placement, conversion to moderate dementia, and death in severe dementia, with crude and adjusted ORs (95\% Cl) of rapid cognitive decline according to quartiles of cerebrospinal fluid total tau

\begin{tabular}{|c|c|c|c|c|c|c|c|c|}
\hline \multirow[t]{2}{*}{$\overline{C S F}$ t-tau (ng/L) } & \multicolumn{2}{|c|}{ Nursing home placement $(n=112 / 234)$} & \multicolumn{2}{|c|}{ Conversion to moderate dementia $(n=149 / 219)$} & \multicolumn{2}{|c|}{ Rapid cognitive decline ${ }^{a}(n=57 / 213)$} & \multicolumn{2}{|c|}{ Death in severe dementia $(n=46 / 234)$} \\
\hline & Crude & Multivariate $^{\mathrm{b}}$ & Crude & Multivariate $^{\mathrm{b}}$ & Crude & Multivariate $^{\mathrm{b}}$ & Crude & Multivariate $^{\mathrm{b}}$ \\
\hline$\geq 900$ & $2.61(1.54-4.42)^{c}$ & $2.17(1.24-3.80)^{c}$ & $2.51(1.59-3.96)^{c}$ & $2.34(1.45-3.77)^{c}$ & $2.91(1.25-6.79)^{c}$ & $3.04(1.16-8.99)^{c}$ & $2.94(1.29-6.68)^{c}$ & $2.64(1.10-6.33)^{c}$ \\
\hline 681-899 & $1.79(1.003-3.21)^{c}$ & $1.72(0.94-3.14)$ & $1.39(0.86-2.23)$ & $1.19(0.72-1.96)$ & $2.05(0.80-5.26)$ & $1.81(0.66-4.94)$ & $1.46(0.56-3.78)$ & $0.94(0.30-2.90)$ \\
\hline $511-680$ & $1.42(0.80-2.51)$ & $1.50(0.82-2.75)$ & $0.90(0.56-1.47)$ & $0.97(0.59-1.59)$ & $0.90(0.34-2.42)$ & $0.88(0.30-2.55)$ & $1.39(0.54-3.61)$ & $1.17(0.42-3.30)$ \\
\hline$\leq 510$ & 1.00 & 1.00 & 1.00 & 1.00 & 1.00 & 1.00 & 1.00 & 1.00 \\
\hline
\end{tabular}

CSF cerebrospinal fluid

${ }^{a} \geq 4$-point decline in Mini Mental State Examination score per 12 months

${ }^{b}$ Multivariate models are adjusted for age, sex, living condition, education level, mild to moderate dementia, and Mini Mental State Examination score at baseline

${ }^{c} p<0.05$ 


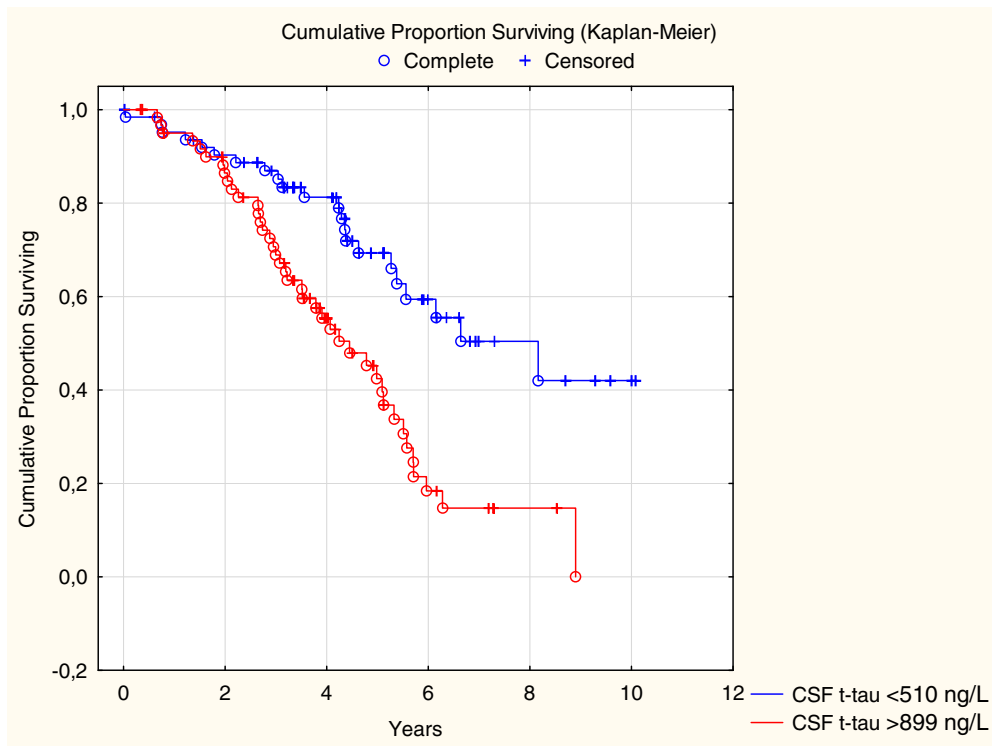

Fig. 2 Cumulative HRs of nursing home placement by baseline levels of cerebrospinal fluid (CSF) total tau (t-tau) (highest quartile compared with the lowest quartile)

in the MCI stage in both familiar and sporadic AD $[27,28]$, and they remain constant thereafter [11]. CSF t-tau is classified as a marker of neuronal injury in the National Institute on Aging-Alzheimer's Association criteria [29]. The magnitude of increase in CSF tau levels is very high in disorders with malignant degeneration, such as Creutzfeldt-Jakob disease [30, 31]. However, in pure tauopathies such as progressive supranuclear paralysis and corticobasal degeneration, which generally have a faster progression than $\mathrm{AD}, \mathrm{t}$-tau in CSF is only moderately increased [32]. This suggests the existence of specific mechanisms behind $\mathrm{AD}$ tauopathy. In addition to a passive release from dying neurons, there is accumulating evidence that tau is actively secreted and transmitted between synaptically connected neurons in a prion-like manner $[12,13]$. Hence, high CSF t-tau in AD may reflect not only a high passive release of aggregated tau from dying neurons but also a high fraction of "active" tau and the intensity of $\mathrm{AD}$ spreading, which also adds support to new therapeutic approaches acting in the extracellular

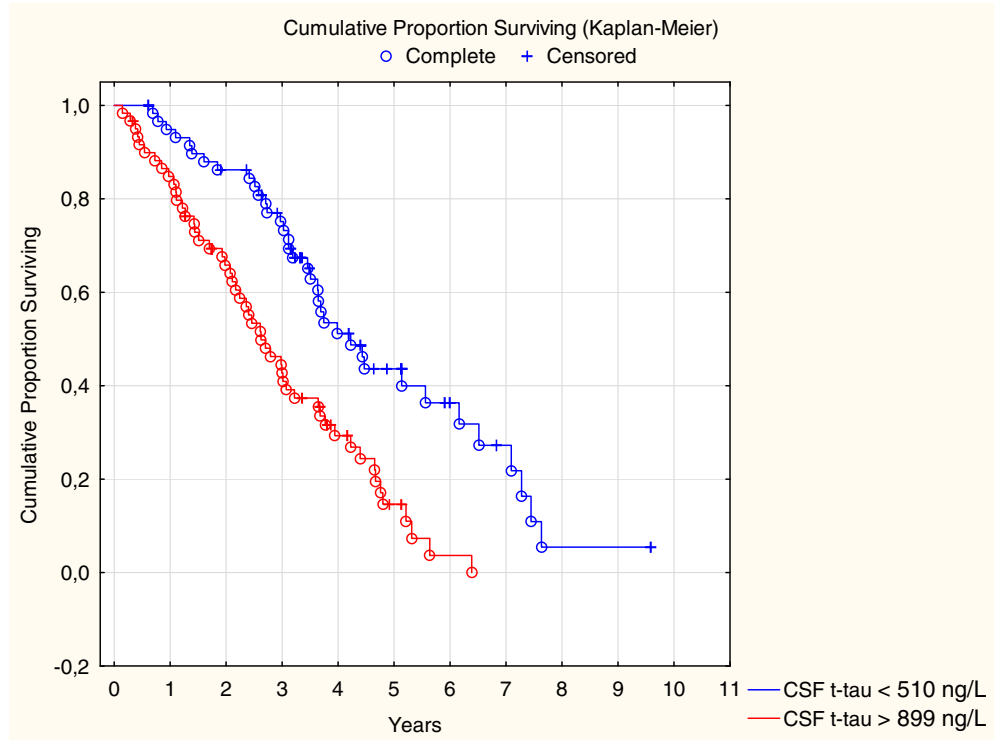

Fig. 3 Cumulative HRs of conversion to moderate dementia by baseline levels of cerebrospinal fluid (CSF) total tau (t-tau) (highest quartile compared with the lowest quartile) 
space [33]. Our finding that high CSF t-tau, rather than p-tau, is associated with AD progression rate is well in concordance with this model.

One of the authors (LK) reviewed all published studies (1756 in November 2015) resulting from a PubMed search using the keywords "Alzheimer's disease," "cerebrospinal fluid," and "tau." Similar to our findings, the vast majority of the cross-sectional studies described no association between tau levels and disease stage. Moreover, most longitudinal studies focused on distinguishing AD from nonprogressive $\mathrm{AD}$ (i.e., non- $\mathrm{AD}$, cases in $\mathrm{MCI}$ populations), where abnormal biomarkers, as expected, predict conversion to AD. Regarding CSF biomarkers and progression in the dementia stage of $\mathrm{AD}$, we found seven longitudinal studies, and the results were contradictory. Two studies with a follow-up period of approximately 3 years showed that high $\mathrm{t}$-tau predicted steeper decline in MMSE score $[22,23]$, but the results from the other five studies were not clear-cut $[34,35]$ or negative [36-38], in this respect, for patients with $\mathrm{AD}$ dementia. The association between CSF biomarkers and loss of ADL has been assessed in two reports from the Alzheimer's Disease Neuroimaging Initiative cohort, with no clear evidence of any relation with high CSF tau in patients who had reached the dementia stage $[39,40]$. No study had NHP or conversion to moderate dementia as outcomes, which likely reflects the lack of health care systems enabling long-term follow-up. Caregiver burden, including loss of PADL and behavioral and psychological symptoms of dementia (BPSD), underlie the decision for NHP, as does the health of the spouse. In patients living alone, potential hazards, together with BPSD and the quality of the home care, are important factors. Interestingly, one report described a relationship between high CSF tau and psychosis in AD [41].

Only a few studies have assessed the relationship with AD mortality. In a small study, high CSF tau was associated with shorter 6-year survival [42]. Cluster analyses of a larger cohort showed a relationship between very high CSF t-tau and low A $\beta 42$ and mortality [34]. In contrast, high tau was associated with shorter survival in dementia with Lewy bodies, but not in AD, in a cohort followed for up to 7 years [43]. Mortality is not an ideal marker of progression, since many patients have a "preterm" death due to non-AD-related causes, which attenuates possible relationships. In a recent study done in southern Sweden with up to 12 years of follow-up [44], researchers found no relationship between high $\mathrm{t}$-tau and early death caused by dementia. However, misdiagnosis cannot be excluded, since data were collected from a registry. In the present study, it was possible to capture "death in severe dementia" by scrutinizing patients' status through the medical records.

CSF analyses have been a part of the diagnostic procedures at Swedish memory clinics since the beginning of the 2000s [2]. Forty percent of 17,000 newly diagnosed patients in the National Swedish Dementia Registry (SveDem; 2007-2011), where $92 \%$ were assessed at a specialist unit, underwent LP [45]. Our cohort is likely to be representative of patients with $\mathrm{AD}$ in the younger age group who consult memory clinics for diagnostic workup. Further, the cohort is probably representative of participants in $\mathrm{AD}$ clinical trials, who are recruited mainly from a memory clinic, and a coliving spouse is generally needed. However, it differs from a general AD population with respect to younger age, higher education ( $21 \%$, reflecting the setting in a university city), and cohabiting status. Twenty-four percent were living alone, which is less than in a general Swedish dementia population, where $47 \%$ live alone at the time of diagnosis (data from SveDem, 50,000 patients, 2007-2015). Patients with $\mathrm{AD}$ living together with a spouse are likely to be diagnosed at an earlier time point than those who live by themselves. Since low education and solitary living are factors associated with early NHP, our results would be an underestimation of the true associations. NHP might be a valid marker of rapid decline in our population, since costs of nursing home care in Sweden are provided by the authorities and are available regardless of income. However, the timing of NHP differs between countries, depending on ethnicity, social structure, and socioeconomic status.

The major strength of this study is the long follow-up period of a large AD cohort, where the diagnostic workup was scrutinized and reevaluated. Virtually all subjects had at least one positive CSF biomarker, and loss to follow-up was minimal. We were able to capture dates of NHP and the approximate time point for conversion to moderate dementia, as well as to evaluate the medical conditions at the time of death. This was possible because of the personal identity numbers assinged to all Swedish citizens and the sharing of digital medical records by all health care units, including the dementia group living facilities. Another strength of the cohort is the clinical setting at a memory clinic rather than a research program with highly selected patients. Time to NHP was chosen as a robust major outcome, and the association between increased CSF t-tau and rapid deterioration was confirmed using three other outcomes reflecting factors of vital importance for patients, caregivers, and societal costs.

The present study also has limitations. One is the lack of systematic follow-up at predefined time points for the cognitive and functional assessments, as well as the lack of formal ADL and BPSD scales. Instead, intervals varied according to the individual patient's needs; for example, assessments of MMSE and ADL were more frequent in rapid decliners. This would only create more contrast between the groups, however. 


\section{Conclusions}

This study provides evidence of a biomarker that already at the stage of MCI due to $\mathrm{AD}$ is a marker of future deterioration rate. Our findings of an association between rapid decline and high CSF t-tau, rather than A $\beta 42$ or $\mathrm{p}$-tau, are in accordance with the understanding of tau as a marker of aggressive neurodegeneration, including active interneuronal tau propagation. Further prospective studies using standardized measurements are needed to confirm the predictive value of t-tau in CSF. During the past few years, research on AD biomarkers has been focused on identifying preclinical cases that may benefit from future disease-halting treatment. However, clinical research ought to focus more on patients with rapid decline, in view of the very low quality of life of patients and caregivers and the high societal costs. Previous immunotherapy studies may have failed in part due to misdiagnosis and/or slower than expected disease progression in the highly selected participants. Clinical trials evaluating the efficacy of potential AD diseasemodifying drugs should take into consideration the large interindividual variation of progression rate and may benefit from selecting participants with high CSF tau levels.

To our knowledge, this is the first study to show that high CSF t-tau levels predict early NHP and conversion to moderate dementia in an $\mathrm{AD}$ cohort. Selecting patients with high CSF $\mathrm{t}$-tau, indicating a more aggressive neurodegeneration and steeper decline, for AD immunotherapy trials might increase the possibility of showing contrast between active treatment and placebo.

\section{Additional files}

Additional file 1: Table S1. Crude and adjusted Cox proportional hazards ratios $(95 \% \mathrm{Cl}$ ) of nursing home placement, conversion to moderate dementia, and death in severe dementia; crude and adjusted OR $(95 \% \mathrm{Cl})$ of rapid cognitive decline according to quartiles of CSF p-tau. (DOCX $15 \mathrm{~kb}$ )

Additional file 2: Table S2. Crude and adjusted Cox proportional hazards ratios $(95 \% \mathrm{Cl})$ of nursing home placement, conversion to moderate dementia, and death in severe dementia; crude and adjusted OR $(95 \% \mathrm{Cl})$ of rapid cognitive decline according to quartiles of CSF amyloid- $\beta_{42}$. (DOCX $\left.15 \mathrm{~kb}\right)$

\footnotetext{
Abbreviations

Aß42: amyloid- $\beta 42$ peptide; AD: Alzheimer's disease; ADL: activities of daily living; BPSD: behavioral and psychological symptoms of dementia; CSF: cerebrospinal fluid; ELISA: enzyme-linked immunosorbent assay; GP: general practitioner; LP: lumbar puncture; MCl: mild cognitive impairment; MMSE: Mini Mental State Examination; NHP: nursing home placement; OD: optical density; PADL: personal activities of daily living; p-tau: phosphorylated tau; SveDem: National Swedish Dementia Registry; t-tau: total tau.
}

\section{Acknowledgments}

The authors thank research nurses Käthe Ström and RoseMarie Brundin for all their assistance in the study.

\section{Funding}

This study was supported by grants from the Swedish Alzheimer Foundation and Medical Training and Research Agreement (ALF) funding from Uppsala University Hospital.

\section{Availability of supporting data}

Raw data are available upon request, but patient journals and laboratory data with patient IDs will not be available due to patient confidentiality requirements.

\section{Authors' contributions}

MDG: study concept and design; active participation in the diagnostic workup of the patients; acquisition, analysis, and interpretation of data; and writing of the manuscript. Ml: study concept and design, critical revision of the manuscript for important intellectual content, and active participation in the diagnostic workup of the patients. KB: head of the clinical neurochemistry laboratory, where the immunoassays of biomarkers were analyzed; critical revision of the manuscript for important intellectual content; and writing of the manuscript. HB: study concept and design, active participation in the diagnostic workup of the patients, and critical revision of the manuscript for important intellectual content. LL: study concept and design, active participation in the diagnostic workup of the patients, and critical revision of the manuscript for important intellectual content. LK: study concept and design, active participation in the diagnostic workup of the patients, literature review, and writing of the manuscript. All authors read and approved the final manuscript.

\section{Competing interests}

The authors declare that they have no competing interests.

Ethical approval and consent to participate

The study was approved by the Uppsala Ethical Review Board, and the patients provided their informed consent.

\section{Author details}

${ }^{1}$ Department of Public Health and Caring Sciences/Geriatrics, Uppsala University, Uppsala Science Park, SE-751 85 Uppsala, Sweden. ${ }^{2}$ Clinical Neurochemistry Laboratory, Institute of Neuroscience and Physiology, University of Gothenburg, Sahlgrenska University Hospital, SE-43180 Mölndal, Sweden.

Received: 17 December 2015 Accepted: 29 April 2016

Published online: 06 June 2016

\section{References}

1. Kua EH, Ho E, Tan HH, Tsoi C, Thng C, Mahendran R. The natural history of dementia. Psychogeriatrics. 2014;14:196-201.

2. Wübker A, Zwakhalen SM, Challis D, Suhonen R, Karlsson S, Zabalequi A, et al. Costs of care for people with dementia just before and after nursing home placement: primary data from eight European countries. Eur J Health Econ. 2015;16:689-707.

3. Holmes C, Lovestone S. Long-term cognitive and functional decline in late onset. Alzheimer's disease: therapeutic implications. Age Aging. 2003;32:200-4

4. Gillette-Guyonnet S, Andrieu S, Nourhashemi F, Gardette V, Coley N, Cantet $C$, et al. Long-term progression of Alzheimer's disease in patients under antidementia drugs. Alzheimers Dement. 2011;7:579-92.

5. Bloom GS. Amyloid- $\beta$ and tau: the trigger and bullet in Alzheimer disease pathogenesis. JAMA Neurol. 2014:71:505-8.

6. Blennow K, Hampel H, Weiner M, Zetterberg $H$. Cerebrospinal fluid and plasma biomarkers in Alzheimer disease. Nat Rev Neurol. 2010;6:131-44

7. Jensen M, Basun H, Lannfelt L. Increased cerebrospinal fluid tau in patients with Alzheimer's disease. Neurosci Lett. 1995;186:189-91.

8. Blennow K, Wallin A, Agren H, Spenger C, Siegfried J, Vanmechelen E. Tau protein in cerebrospinal fluid: a biochemical marker for axonal degeneration in Alzheimer's disease? Mol Chem Neuropathol. 1995;26:231-45.

9. Parnetti L, Chiasserini D, Eusebi P, Giannandrea D, Bellomo G, De Carlo C, et al. Performance of $A \beta_{1-40}, A \beta_{1-42}$, total tau, and phosphorylated tau as predictors of dementia in a cohort of patients with mild cognitive impairment. J Alzheimers Dis. 2012;29:229-38. 
10. van Rossum IA, Vos SJB, Burns $L$, Knol DL, Scheltens $P$, Soininen $H$, et al. Injury markers predict cognitive decline in subjects with $\mathrm{MCl}$ and amyloid pathology. Neurology. 2012;79:1809-16.

11. Mattsson N, Portelius E, Rolstad S, Gustavsson M, Andreasson U, Stridsberg $M$, et al. Longitudinal cerebrospinal fluid biomarkers over four years in mild cognitive impairment. J Alzheimers Dis. 2012;30:767-78.

12. Pooler AM, Polydoro M, Wegmann S, Nicholls SB, Spires-Jones TL, Hyman BT. Propagation of tau pathology in Alzheimer's disease: identification of novel therapeutic targets. Alzheimers Res Ther. 2013;5:49.

13. Sanders DW, Kaufman SK, DeVos SL, Sharma AM, Mirbaha H, Li A, et al. Distinct tau prion strains propagate in cells and mice and define different tauopathies. Neuron. 2014;82:1271-88.

14. Kontsekova E, Zilka N, Kovacech B, Novak P, Novak M. First-in-man tau vaccine targeting structural determinants essential for pathological tau-tau interaction reduces tau oligomerisation and neurofibrillary degeneration in an Alzheimer's disease model. Alzheimers Res Ther. 2014;6:44.

15. Degerman Gunnarsson $M$, Lannfelt L, Ingelsson M, Basun $H$, Kilander L. High tau levels in cerebrospinal fluid predict rapid decline and increased dementia mortality in Alzheimer's disease. Dement Geriatr Cogn Disord. 2014;37:196-206.

16. Folstein MF, Folstein SE, McHugh PR. "Mini-mental state". A practical method for grading the cognitive state of patients for the clinician. J Psychiatr Res. 1975;12:189-98.

17. McKhann G, Drachman D, Folstein M, Katzman R, Price D, Stadlan EM. Clinical diagnosis of Alzheimer's disease: report of the NINCDS-ADRDA Work Group under the auspices of Department of Health and Human Services Task Force on Alzheimer's disease. Neurology. 1984;34:939-44.

18. American Psychiatric Association. Diagnostic and statistical manual of mental disorders. 4th ed. Washington, DC: American Psychiatric Association; 1994

19. Vanmechelen $E$, Vanderstichele $H$, Davidsson $P$, Van Kerschaver E, Van Der Perre $B$, Sjögren $M$, et al. Quantification of tau phosphorylated at threonine 181 in human cerebrospinal fluid: a sandwich ELISA with a synthetic phosphopeptide for standardization. Neurosci Lett. 2000;285:49-52.

20. Andreasen N, Hesse C, Davidsson P, Minthon L, Wallin A, Winblad B, et al. Cerebrospinal fluid $\beta$-amyloid ${ }_{(1-42)}$ in Alzheimer disease: differences between early- and late-onset Alzheimer disease and stability during the course of disease. Arch Neurol. 1999:56:673-80.

21. Winblad B, Palmer K, Kivipelto M, Jelic V, Fratiglioni L, Wahlund LO, et al. Mild cognitive impairment - beyond controversies, towards a consensus: report of the International Working Group on Mild Cognitive Impairment. J Intern Med. 2004;256:240-6.

22. Kester MI, van der Vlies AE, Blankenstein MA, Pijnenburg YA, van Elk EJ, Scheltens $P$, et al. CSF biomarkers predict rate of cognitive decline in Alzheimer disease. Neurology. 2009;73:1353-8.

23. Sämgård K, Zetterberg H, Blennow K, Hansson O, Minthon L, Londos E. Cerebrospinal fluid total tau as a marker of Alzheimer's disease intensity. Int J Geriatr Psychiatry. 2010;25:403-10.

24. Jack CR Jr, Knopman DS, Jagust WJ, Petersen RC, Weiner MW, Aisen PS, et al. Tracking pathophysiological processes in Alzheimer's disease: an updated hypothetical model of dynamic biomarkers. Lancet Neurol. 2013;12:207-16.

25. Ingelsson M, Fukumoto $H$, Newell KL, Growdon JH, Hedley-Whyte ET, Frosch MP, et al. Early A $\beta$ accumulation and progressive synaptic loss, gliosis, and tangle formation in AD brain. Neurology. 2004;62:925-31.

26. Nelson PT, Alafuzoff I, Bigio EH, Bouras C, Braak H, Cairns NJ, et al. Correlation of Alzheimer disease neuropathologic changes with cognitive status: a review of the literature. J Neuropathol Exp Neurol. 2012;71:362-81.

27. Fleisher AS, Chen K, Quiroz YT, Jakimovich LJ, Gutierrez Gomez M, Langois $\mathrm{CM}$, et al. Associations between biomarkers and age in the presenilin 1 E280A autosomal dominant Alzheimer disease kindred: a cross-sectional study. JAMA Neurol. 2015;72:316-24.

28. Bertens D, Knol DL, Scheltens P, Visser PJ, Alzheimer's Disease Neuroimaging Initiative. Temporal evolution of biomarkers and cognitive markers in the asymptomatic, $\mathrm{MCl}$, and dementia stage of Alzheimer's disease. Alzheimers Dement. 2015:11:511-22.

29. McKhann GM, Knopman DS, Chertkow H, Hyman BT, Jack CR Jr, Kawas CH et al. The diagnosis of dementia due to Alzheimer's disease: recommendations from the National Institute on Aging-Alzheimer's Association workgroups on diagnostic guidelines for Alzheimer's disease. Alzheimers Dement. 2011;7:263-9.
30. Skillbäck T, Rosén C, Asztely F, Mattsson N, Blennow K, Zetterberg H. Diagnostic performance of cerebrospinal fluid total tau and phosphorylated tau in Creutzfeldt-Jakob disease: results from the Swedish Mortality Registry. JAMA Neurol. 2014:71:476-83.

31. Lewczuk P, Mroczko B, Fagan A, Kornhuber J. Biomarkers of Alzheimer's disease and mild cognitive impairment: a current perspective. Adv Med Sci. 2015;60:76-82.

32. Hall S, Öhrfelt A, Constantinescu R, Andreasson U, Surova Y, Bostrom F, et al. Accuracy of a panel of 5 cerebrospinal fluid biomarkers in the differential diagnostics of patients with dementia and/or Parkinsonian disorders. Arch Neurol. 2012:69:1445-52.

33. Holmes BB, Diamond MI. Prion-like properties of tau protein: the importance of extracellular tau as a therapeutic target. J Biol Chem. 2014;289:19855-61.

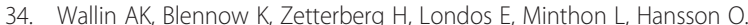
CSF biomarkers predict a more malignant outcome in Alzheimer disease. Neurology. 2010;11:1531-7.

35. Seppälä TT, Koivisto AM, Hartikainen P, Helisalmi S, Soininen H, Herukka SK. Longitudinal changes of CSF biomarkers in Alzheimer's disease. J Alzheimers Dis. 2011:25:583-94

36. Vlachos GS, Paraskevas GP, Naoumis D, Kapaki E. Cerebrospinal fluid $\beta$-amyloid ${ }_{1-42}$ correlates with rate of progression in Alzheimer's disease J Neural Transm. 2012;119:799-804

37. Vemuri $\mathrm{P}$, Wiste HJ, Weigand SD, Shaw LM, Trojanowski JQ, Weiner MW et al. MRI and CSF biomarkers in normal, MCl, and AD subjects: predicting future clinical change. Neurology. 2009;28:294-301.

38. Sluimer JD, Bouwman FH, Vrenken H, Blankenstein MA, Barkhof F, van der Flier WM, et al. Whole-brain atrophy rate and CSF biomarker levels in $\mathrm{MCl}$ and AD: a longitudinal study. Neurobiol Aging. 2010;31:758-64.

39. Okonkwo OC, Alosco ML, Jerskey BA, Sweet LH, Ott BR, Tremont G, et al. Cerebrospinal fluid abnormalities and rate of decline in everyday function across the dementia spectrum: normal aging, mild cognitive impairment, and Alzheimer disease. Arch Neurol. 2010;67:688-96.

40. Marshall GA, Lorius N, Locascio JJ, Hyman BT, Rentz DM, Johnson KA, et al. Regional cortical thinning and cerebrospinal biomarkers predict worsening daily functioning across the Alzheimer's disease spectrum. J Alzheimers Dis. 2014;41:719-28.

41. Koppel J, Sunday S, Buthorn J, Goldberg T, Davies P, Greenwald B, et al. Elevated CSF tau is associated with psychosis in Alzheimer's disease. Am J Psychiatry. 2013;170:1212-3.

42. Wallin AK, Blennow K, Andreasen N, Minthon L. CSF biomarkers for Alzheimer's disease: levels of $\beta$-amyloid, tau, phosphorylated tau relate to clinical symptoms and survival. Dement Geriatr Cogn Disord. 2006;21:131-8

43. Boström F, Hansson O, Blennow K, Gerhardsson L, Lundh T, Minthon L, et al. Cerebrospinal fluid total tau is associated with shorter survival in dementia with Lewy bodies. Dement Geriatr Cogn Disord. 2009;28:314-9.

44. Nägga K, Wattmo C, Zhang Y, Wahlund LO, Palmqvist S. Cerebral inflammation is an underlying mechanism of early death in Alzheimer's disease: a 13-year cause-specific multivariate mortality study. Alzheimers Res Ther. 2014:6:41.

45. Falahati F, Fereshtehnejad SM, Religa D, Wahlund LO, Westman E, Eriksdotter M. The use of MRI, $C T$ and lumbar puncture in dementia diagnostics: data from the SveDem registry. Dement Geriatr Cogn Disord. 2015;39:81-91.

\section{Submit your next manuscript to BioMed Central and we will help you at every step:}

- We accept pre-submission inquiries

- Our selector tool helps you to find the most relevant journal

- We provide round the clock customer support

- Convenient online submission

- Thorough peer review

- Inclusion in PubMed and all major indexing services

- Maximum visibility for your research

Submit your manuscript at www.biomedcentral.com/submit 